Accepted for publication in IEEE Transactions on Plasma Science

November 2010

\title{
Origin of the Delayed Current Onset in High Power Impulse Magnetron Sputtering
}

\author{
Georgy Yu. Yushkov ${ }^{1}$ and André Anders ${ }^{2 *}$, Fellow IEEE \\ ${ }^{1}$ High Current Electronics Institute, Russian Academy of Sciences, Tomsk, 634055, Russia; \\ ${ }^{2}$ Lawrence Berkeley National Laboratory, Berkeley, California 94720, USA. \\ *corresponding author: aanders@lbl.gov
}

Revised version, July 19, 2010

\begin{abstract}
DISCLAIMER
This document was prepared as an account of work sponsored by the United States Government. While this document is believed to contain correct information, neither the United States Government nor any agency thereof, nor The Regents of the University of California, nor any of their employees, makes any warranty, express or implied, or assumes any legal responsibility for the accuracy, completeness, or usefulness of any information, apparatus, product, or process disclosed, or represents that its use would not infringe privately owned rights. Reference herein to any specific commercial product, process, or service by its trade name, trademark, manufacturer, or otherwise, does not necessarily constitute or imply its endorsement, recommendation, or favoring by the United States Government or any agency thereof, or The Regents of the University of California. The views and opinions of authors expressed herein do not necessarily state or reflect those of the United States Government or any agency thereof or The Regents of the University of California.
\end{abstract}

This work was supported by the U.S. Department of Energy, Initiatives for Proliferation Prevention, under Contract No. DE-AC02-05CH11231 with the Lawrence Berkeley National Laboratory. 


\title{
Origin of the Delayed Current Onset in High Power Impulse Magnetron Sputtering \\ Georgy Yu. Yushkov and André Anders, Fellow IEEE
}

\begin{abstract}
Repetitive pulses of voltage and current are applied in high power impulse magnetron sputtering. The current pulse usually lags the applied voltage by a significant time, which in some cases can reach many 10s of microseconds. The current time lag is generally highly reproducible and jitters less than $1 \%$ of the delay time. This work investigates the time lag experimentally and theoretically. The experiments include several different target and gas combinations, voltage and current amplitudes, gas pressures, pulse repetition rates, and pulse durations. It is shown that in all cases the inverse delay is approximately proportional to the applied voltage, where the proportionality factor depends on the combination of materials and the conditions selected. The proportionality factor contains the parameters of ionization and secondary electron emission. The statistical time lag is negligible while the formative time lag is large and usually dominated by the ion motion (inertia), although, at low pressure, the long free path of magnetized electrons causing ionization contributes to the delay.
\end{abstract}

\section{INTRODUCTION}

High power impulse magnetron sputtering (HIPIMS) is a rapidly developing coatings technology that combines traditional magnetron sputtering with pulsed power technology [1-2]. The main objective of using high power density is to ionize the sputtered atoms, which gives us the opportunity to better tailor the properties of coatings via self-ion assistance, i.e. assistance by the ions that condense and contribute to film growth. As a result, dense, well textured films can be obtained that are often superior to films deposited by evaporation or conventional sputtering.

Even in the early, seminal paper by Kouznetsov et al. [1], a very significant delay of the current onset was shown in their Fig. 1: it took about $50 \mu$ s (a very long time!) after the voltage of $1.5 \mathrm{kV}$ was applied before any noticeable discharge current was recorded. Virtually all researchers have seen some kind of noticeable or significant current delay in their setups. However, not much is said about this delay even as it clearly affects the instantaneous and average power, the plasma properties, and indirectly the film properties. One reason for the silence is perhaps that the delay is exceptionally reproducible. One can simply accept its existence and adjust the parameters such as pulse length accordingly.

However, it is interesting to ask: Why does this delay exist? And why is it so well reproducible? These are the questions that are going to be addressed in this contribution. In the experimental section, the delayed onset will be quantified for a range of conditions. We will show that the observed delays are compatible with theoretical considerations done for electrical breakdown and the onset of gas discharges, which have been investigated many years even before the invention of the sputtering magnetron.

\section{EXPERIMENTAL SETUP}

A planar magnetron with a 2-inch $(25 \mathrm{~mm})$ diameter disk target was mounted in a cryogenically pumped high vacuum chamber. New targets with a nominal (i.e. before 
erosion) thickness of a $1 / 4$ inch $(6.25 \mathrm{~mm})$ thick were used. The following pure metals were used in this study: $\mathrm{Zn}, \mathrm{Cu}, \mathrm{Nb}, \mathrm{Cr}$ and $\mathrm{W}$. After use, the targets showed the familiar erosion ring (known as the "racetrack") of about $25 \mathrm{~mm}$ diameter. The magnetron was approximately balanced with the highest magnetic induction in the center of the target being about $60 \mathrm{mT}$. The base pressure of the system was approximately $1 \times 10^{-4} \mathrm{~Pa}$ as determined by an ion gauge. Research grade argon gas was introduced via a calibrated mass flow controller at a flow rate of $50 \mathrm{sccm}$; the process pressure could be adjusted by reducing the maximum pumping speed of $1500 \mathrm{l} / \mathrm{s}$ by using an adjustable gate valve. The total process pressure was monitored by an MKS Baratron ${ }^{\mathbb{B}}$ capacitance manometer.

The magnetron was connected to a HIPIMS power supply: we used an upgraded SPIK2000A by Melec capable of constant voltage pulses with a current of up to $500 \mathrm{~A}$. The voltage pulse duration could be adjusted but here we fixed the duration to $400 \mu \mathrm{s}$ with the exception of one experiment when the effect of the pulse length was investigated. The relatively long time of $400 \mu$ s allows the discharge to develop from the initial, gas-dominated discharge phase to a phase where self-sputtering is important, should the target material and other conditions favor this development. It also allowed us to accommodate very long delay times in this study. The magnetron is designed for a maximum average power of $1 \mathrm{~kW}$, and therefore the pulse repetition rate must not be too high. The simplest way of making sure that the power limit is not exceeded is to limit the power delivered by the SPIK's charging power supply (Pinnacle ${ }^{\circledR}$ by Advanced Energy).

The time dependent current and voltage pulses were measured with a fast, widebandwidth Pearson ${ }^{\mathrm{TM}}$ current transformer (model 301X, $20 \mathrm{MHz}$ ) and a 100:1 voltage divider (Tektronix P5100, $250 \mathrm{MHz}$ bandwidth), respectively. The voltage divider was connected to the target (at the cathode feedthrough), while the magnetron anode was at ground potential. The data were recorded with a digital oscilloscope (Tektronix TDS 5054B) and exported to a personal computer for further analysis.

\section{RESULTS}

The first experiment shows that the delay between voltage application (Fig. 1, top) and current onset (Fig. 1, bottom) can be very large, and for certain conditions may even exceed $100 \mu \mathrm{s}$, which is comparable to typical pulse lengths used in HIPIMS. Figure 1 also illustrates the well-known fact that the delay is a function of the applied voltage. Results like this, here with a copper target, are surprisingly reproducible. Using a different target, niobium, at a relatively low voltage, we can find again that the onset is much delayed. Notably, the jitter of current onset is less than $0.5 \%$. Note that the time "zero" in Fig. 2 is defined by the time of voltage application, i.e. we have "zoomed" into the relevant time window of current onset. In the next two figures, Figs. 3 and 4, the current onset is show as a function of the applied voltage, with the target material and the process gas as parameters.

We comment that the current is lower than we were used to from our previous HIPIMS experiments [3]. Most experiments were done with voltages close to the threshold of discharge existence, hence with low currents. Additionally, the use of new targets still having their full nominal thickness implies having a relatively weak magnetic surface field. As the experiments progressed we found a gradual shift to higher and higher currents. Since we are concerned with the onset of the discharge we do not further address those shifts here. 
So far, the process gas pressure was kept constant at $1.3 \mathrm{~Pa}$. Fig. 5 shows the slight changes when the pressure is changed: the top presentation is the familiar display with the applied voltage as the variable, for a zinc target and with the pressure as a parameter, and the bottom presentation uses the pressure as the variable when a copper target is used.

Other parameters of HIPIMS processing include the pulse duration and the pulse repetition rate. Therefore, also these variables were included in this study. From Fig. 6 we can see, specifically for the tungsten-target argon-gas combination, that the current delay is not further reduced once the pulse duration is longer than about $150 \mu \mathrm{s}$, or the repetition rate is greater than 150 pulses per second.

\section{DISCUSSION}

The onset of a discharge has been extensively investigated in the late $19^{\text {th }}$ century and first half of the $20^{\text {th }}$ century - we just remind the reader about the well-known Paschen curves [4], which relate the minimum breakdown voltage to the product of gas pressure and electrode distance, Townsend's law on avalanche development [5], the role of metastable gas atoms discovered by Penning [6-7], and the minimum of the ionization cross section at very low electron energies - the quantum-mechanical Ramsauer effect [8].

The main difference to the early work is that we consider a magnetron configuration, where electron motion is governed by a non-uniform magnetic field with well-known $\mathbf{E} \times \mathbf{B}$ drift effects.

Following the early concepts of breakdown, the delay of current onset, also known as time lag, is composed of two contributions [5, 9]: (i) the statistical time lag, $t_{s}$, for initial electrons to appear in the electric field between electrodes, and (ii) the formative time lag, $t_{f}$, corresponding to the time required for the discharge to develop from the initial electrons. Hence the total delay is

$$
t_{d}=t_{s}+t_{f} \text {. }
$$

This concept is widely accepted [9-13]. The statistical time lag can be much larger than the formative time lag if the study is done very close to the static breakdown voltage, $V_{0}[9,12]$. Any voltage greater than $V_{0}$ is called an overvoltage. Pulsed systems generally operate with large overvoltage in order to quickly and reliably obtain a discharge. For HIPIMS and the lower current, mid-frequency pulsed sputtering, the jitter is very small and therefore the statistical lag is small:

$$
t_{s} \ll t_{f} \text {. }
$$

The special case of glow discharge onset with noble gases has been intensely studied in the 1930s [14-16]. Following Schade's classical analysis [15], we consider the case where initial electrons are present (e.g., left over from a previous discharge). Upon application of a voltage $V$, the electrons will be accelerated to the anode by the electric field. In our special case of a magnetron, the motion of electrons is impeded by the magnetic field. For most electrons, the local magnetic field vector will not be perfectly perpendicular to the electric vector. The parallel component $\mathbf{E}_{\|}$allows the electrons to pick up energy which can exceed the ionization energy of the gas atoms (metal atoms at a later stage). Using the common Townsend nomenclature, the ionization by electron 
impact can be described by the ionization coefficient $\alpha$, the number of ionization events per path length. When an ion is created by electron impact, the electric field will accelerate it to the target (cathode). We note that at this stage of the discharge, no sheath (space charge layer) has yet formed and the electric field is equally distributed between anode and cathode.

Ion impact on the target generates secondary electrons with a yield $\gamma$. If a secondary electron is produced at a location were the magnetic induction $\mathbf{B}$ is perfectly perpendicular to $\mathbf{E}$, the emitted electron will execute half of a gyration and return to the cathode. In all other cases, the secondary electron can escape from the target and may pickup sufficient energy that can cause ionization. The path of the electron can be rather complicated due to the action of both $\mathbf{B}$ and $\mathbf{E}$, which are not uniform. The path generally resembles a bent helix with superimposed drift components caused by $\mathbf{E} \times \mathbf{B}$, $\nabla \mathbf{B} \times \mathbf{B}$, and more complicated secondary effects [17]. The long path of the electron near the target compensates for the fact the gas density in the magnetron case is much lower than in classical glow discharges.

Let us designate the initial electron current from the target region with $I_{0}$, and the current of secondary electron caused by ions impact at the target with $I_{S E}=\gamma I_{i}$, and the current of ions established in some distance from the target as $I_{i}$. The electron current from the target can be written as

$$
I_{e}(t)=I_{0}+\gamma I_{i}\left(t-t_{i}\right) .
$$

where $t_{i}$ is the average time an ion needs to move from the location of its creation (ionization) to the target surface. The ion current, in turn, is a function of the electron current causing ionization, hence

$$
I_{e}(t)=I_{0}+M I_{e}\left(t-t_{\Delta}\right),
$$

where

$$
t_{\Delta}=t_{i}+t_{e},
$$

is composed of the characteristic ion travel time plus the average time needed by a newly released secondary electron to cause ionization. Furthermore,

$$
M=\gamma[\exp (\alpha d)-1]
$$

is the Townsend multiplication factor resulting from electron impact ionization (the $\alpha$ process) and secondary electron emission (the $\gamma$ process), $d$ is the path length traveled by the electron. The system runs away for $M>1$. The threshold $M=1$ is known as the Townsend breakdown condition. With the generation of many charged particles, a plasma develops. The originally evenly distributed electric field is mostly "pushed out" of the plasma and the voltage drop is largely accommodated by a space charge layer (sheath) adjacent to the target.

We can estimate the characteristic ion and electron travel times as follows. At the low pressures typical for a magnetron $(\sim 1 \mathrm{~Pa})$, the ion most likely can reach the target without collisions and on a relatively straight path because the magnetic field is not strong enough to cause ion magnetization (the ion gyration radius is large compared to the system size). If we furthermore use the fact that a sheath has not yet formed, the ion motion can be treated as uniformly accelerated, leading to 


$$
t_{i}=\sqrt{\frac{2 s}{E} \frac{m_{i}}{q_{i}}},
$$

where $s$ is a characteristic distance from the location of ionization to the nearest target surface, $E$ is the electric field along $s$, and $m_{i}$ and $q_{i}$ are the mass and charge of the ion, respectively. For example, if we consider the formation of an argon ion in $s \sim 1 \mathrm{~cm}$ distance from the target, where the potential of the ionization location is $300 \mathrm{~V}$ above the target potential, the ion will take about $520 \mathrm{~ns}$ to reach the target where it produces the next generation of secondary electrons.

Now we will have a look at the electron timing. The mean free path of an electron before an ionizing collision is

$$
\lambda_{e}=\left(\sigma_{e a}\left(E_{e}\right) n_{a}\right)^{-1}=\left(\sigma_{e a}\left(E_{e}\right) p / k T_{a}\right)^{-1}
$$

corresponding to a characteristic time,

$$
t_{e} \approx \frac{\lambda_{e}}{v_{e}}=\left(\frac{m_{e}}{2 E_{e}}\right)^{1 / 2} \frac{k T_{a}}{\sigma_{e a}\left(E_{e}\right) p},
$$

where $E_{e}$ is a characteristic energy gained by the electron from the electric field, corresponding to a characteristic electron velocity $v_{e} ; m_{e}$ is the electron mass, $T_{a} \approx 300 \mathrm{~K}$ is the gas temperature, $k$ is the Boltzmann constant, $p$ is the pressure, and $\sigma_{e a}\left(E_{e}\right)$ is the ionization cross section which depends on the relative velocity of the electron and atom, i.e. essentially on the electron energy. Let us assume that some of electrons pick up more energy than the ionization energy ( $15.76 \mathrm{eV}$ for argon) and reach the energy region of 50 $-80 \mathrm{eV}$ before the curved magnetic field in conjunction with the local electric field cause them to decelerate, lose energy, and eventually reverse direction. The ionization cross section for argon at the energy $50-80 \mathrm{eV}$ is about $2.7 \times 10^{-20} \mathrm{~m}^{2}$ [18]. If we further consider the low pressure typical for magnetron operation, $p \sim 1 \mathrm{~Pa}$, electrons have to travel a relatively long path $d=\lambda_{e} \approx 0.15 \mathrm{~m}$ for a time of about $t_{e} \approx 30 \mathrm{~ns}$. This time is shorter, but only by about one order of magnitude, than the ion transient time $t_{i}$. Actually, most of the time the electrons will spend at a lower velocity near the turning points of their convoluted path. The cross section at lower energy is lower and becomes zero at the ionization threshold. Considering lower electron energies of about $30 \mathrm{eV}, t_{e}$ increases to about 120 ns. If we furthermore drop the pressure closer to the lowest possible, $p \approx 0.1 \mathrm{~Pa}, t_{e}$ grows to $1.2 \mu$ s and we suddenly deal with a situation were the characteristic electron time may exceed the ion transient time. The conclusion of this discussion is that generally both ion and electron times should be taken into account when calculating the formative time lag. The cycle time $t_{\Delta}$, equation (5), is not the delay for the observable current onset but a key factor showing that the delay is related to the inertia of ions as well as to the long, convoluted path electrons need to travel before an ionizing collision occurs.

The total delay of current onset can be calculated by re-writing (4) as a differential equation [15]

$$
I_{e}(t)=I_{0}+M\left[I_{e}(t)-t_{\Delta} \frac{d}{d t} I_{e}(t)\right]
$$


the solution of which is the formative time lag

$$
t_{f}=\frac{M t_{\Delta}}{M-1} \ln \frac{1+(M-1) I_{e}\left(t_{f}\right) / I_{0}}{M} .
$$

This suggests introducing $\varepsilon=M-1$ as a parameter that shows how much the system is exceeding the breakdown condition. At the threshold, $M=1$ and $\varepsilon=0$, the formative time lag is infinitely long. For small $\varepsilon$, an approximate solution is

$$
t_{f}=\frac{t_{\Delta}}{\varepsilon} \ln \left[1+\varepsilon \frac{I_{e}\left(t_{f}\right)}{I_{0}}\right] .
$$

With the definition (6) we see that $\varepsilon$ is a function of the ionization $(\alpha)$ and secondary electron emission $(\gamma)$ processes, where $\alpha$ depends on the applied voltage. The yield $\gamma$ is a function of the target material and surface conditions but is practically independent of the kinetic energy of the impacting ion (i.e. voltage) [19]. With those assumptions and a Townsend form of the ionization coefficient $[9,13]$

$$
\alpha=A p \exp \left(-\frac{B}{(E / p)}\right)=A p \exp \left(-\frac{B p d}{V}\right),
$$

which for constant pressure can be written as

$$
\alpha=a \exp \left(-\frac{b}{V}\right)
$$

the formative time lag (12) can finally be approximated by [15]

$$
t_{f} \approx \frac{a}{V-V_{0}} \exp \left(\frac{b}{V}\right) .
$$

Here, $A, B, a$, and $b$ are constants (and we note that there is no minus in the exponential factor). In the limiting case of small $\varepsilon$, which means that the applied voltage $V$ is not very much higher than the threshold voltage $V_{0}$, the time lag $t_{f}$ as described by (15) is largely dependent on the pre-exponential factor:

$$
t_{f}^{-1} \propto V-V_{0} .
$$

In the other case, the exponential factor may be developed into a Taylor series $\exp (b / V)=1+b / V+b^{2} / 2 V^{2}+\ldots$, and the non-linear terms can be neglected when the applied voltage is large, $V \gg b$, again resulting a relationship of the type (16).

To prove this rather simple relation, we re-plot our results such that the inverse delay is a function of the applied voltage (Figs. 7). We point out that the assumptions made, like (13), are not proven for the case of very low pressure $p$ and long electron path $d$ as applicable to a magnetron. Very careful observation shows that there are changes of slope within each curve. This is for example visible near the threshold for the $\mathrm{Nb}-\mathrm{Ar}$ combination (Fig. 7 top and center). Clearly, more sophisticated simulations are needed to fully interpret the experimental results.

Looking at Figures 7-9, we can make several conclusions. (i) the threshold voltage can readily be determined by the intersection of the fit curve with the $t_{f}^{-1}=0$ line; (ii) the threshold and the slope of the fit curves are target material dependent, which indicates that the secondary electron emission is clearly important; (iii) the threshold and 
the slope of the fit curves are gas dependent, which indicates that the ionization processes as well as target surface conditions are important; (iv) an increase in gas pressure has almost no influence on the threshold voltage but higher pressure reduces the formative time lag. The latter can be understood because electrons can cause ionizing collisions earlier, and the ionization may be closer to the target when the pressure is high, hence $t_{\Delta}$ shorter.

Unfortunately, the slope is affected by both ionization and secondary emission processes, and the interpretation is therefore convoluted.

Finally, we should mention that $\gamma$ processes other than by ion impact are possible but they are unlikely for the conditions under consideration. For high pressure discharges, and especially for atmospheric pressure conditions, secondary electrons are known to be produced by photoemission rather than by ion impact $[10,13]$. The basic cyclic time is then determined by electron inertia, and the formative time lag is shorter. The observed long lag times a posteriori justify the assumption that ionization avalanche cycles include the motion of ions causing secondary electrons.

The description given so far can explain the sudden yet delayed onset of the discharge current. The amplifying ionization avalanches lead to an increase in current from $I_{0}$ to $I_{e}\left(t_{f}\right)$, which may span as many as 10 orders of magnitude. In our linear presentation of current, like in Figs. 1 and 2, derived from oscilloscope data, we see only the last 1 or 2 orders of the evolution before the current reaches its maximum. The term "onset" is therefore misleading in the sense that it implies no current before the "onset". However, the built-up time, or formative time lag, involves currents which are orders of magnitude smaller than we can see.

The interpretation of the data can also explain the much shorter formative time lag for medium frequency sputtering, or the situation when a low current discharge is sustained between HIPIMS pulses. In those cases, the initial current $I_{0}$ is relatively large, i.e. orders of magnitude higher than at the beginning of low-duty-cycle HIPIMS pulses. The discharge can readily enter a current level detectable by the measuring circuit.

\section{CONCLUSIONS}

The highly reproducible delay of the current pulse indicates that the statistical time lag is negligible most likely because the previous pulse left a sufficiently large number of electrons behind. Those electrons serve as the initial current $I_{0}$, i.e. the seed for ionization avalanches and cycles. The presence of the magnetron's magnetic field modifies the Townsend model of breakdown and discharge onset in that the electron paths are not along the electric field but governed by the combined electric and magnetic field $(\mathbf{E} \times \mathbf{B}$ situation). However, the ions are not magnetized and can reach the target surface essentially following the electric field. The complete delay times can be determined by modifying Schade's approach for the formative time taking into account electron magnetization and delays of electron impact ionization. The formative time lag describes the time needed for ionization avalanches to grow and ionization cycles to complete, which is a well defined process showing little jitter. A simplified evaluation of these processes suggests an inverse relationship between delay time and applied voltage, which is indeed observed for all target material - gas combinations. 


\section{ACKNOWLEDMENTS}

This work was supported by the U.S. Department of Energy, Initiatives for Proliferation Prevention, under Contract No. DE-AC02-05CH11231 with the Lawrence Berkeley National Laboratory. 


\section{References}

[1] V. Kouznetsov, K. Macak, J. M. Schneider, U. Helmersson, and I. Petrov, "A novel pulsed magnetron sputter technique utilizing very high target power densities," Surf. Coat. Technol., vol. 122, pp. 290-293, 1999.

[2] A. Ehiasarian, "Fundamentals and applications of HIPIMS," in Plasma Surface Engineering Research and its Practical Applications, R. Wei, Ed., ed Kerala, India: Research Signpost, 2008, pp. 35-86.

[3] A. Anders, J. Andersson, and A. Ehiasarian, "High power impulse magnetron sputtering: Current-voltage-time characteristics indicate the onset of sustained self-sputtering," J. Appl. Phys., vol. 102, pp. 113303-1-11, 2007.

[4] F. Paschen, "Ueber die zum Funkenübergang in Luft, Wasserstoff und Kohlensäure bei verschiedenen Drucken erforderliche Potentialdifferenz," Wiedemann's Annalen der Physik und Chemie (Neue Serie), vol. 37, pp. 69-96, 1889.

[5] J. S. Townsend, Electricity in Gases. Oxford, 1914.

[6] F. M. Penning, "The starting potential of the glow discharge in neon argon mixtures between large parallel plates: II. Discussion of the ionisation and excitation by electrons and metastable atoms," Physica, vol. 1, pp. 1028-1044, 1934.

[7] A. A. Kruithof and F. M. Penning, "Determination of the Townsend ionization coefficient $\alpha$ for pure argon " Physica, vol. 3, pp. 515-533, 1936.

[8] C. Ramsauer and R. Kollath, Ann. Phys. (Leipzig), vol. 12, p. 536, 1932.

[9] J. M. Meek and J. D. Craggs, Electrical Breakdown of Gases. Oxford, UK: Clarendon Press, 1953.

[10] F. Llewellyn-Jones, Ionization and Breakdown in Gases. London: Methuen \& Co., 1957.

[11] H. M. P. Stock, H. A. Blevin, and J. Fletcher, "Prebreakdown current growth in crossed electric and magnetic fields at low gas pressures: III. Temporal growth of current," J. Phys. D: Appl. Phys., vol. 7, pp. 635-646, 1974.

[12] C. A. Maluckov, J. P. Karamarkovic, and M. K. Radovic, "Statistical analysis of electrical breakdown time delay distributions in neon tube at 13.3 mbar," IEEE Trans. Plasma Sci., vol. 31, pp. 1344-1348, 2003.

[13] Y. P. Raizer, Gas Discharge Physics. Berlin: Springer, 1991.

[14] M. Steenbeck, "The transient course of the ignition of a glow discharge," Wiss. Veröff. Siemens-Konzern, vol. 9, pp. 42-72, 1930.

[15] R. Schade, "Über die Aufbauzeit einer Glimmentladung," Zeitschrift für Physik, vol. 104, pp. 487-510, 1937.

[16] M. J. Druyvesteyn and F. M. Penning, "The mechanism of electrical discharges in gases of low pressure," Rev. Mod. Phys., vol. 12, pp. 87-174, 1940.

[17] F. F. Chen, Plasma Physics and Controlled Fusion. New York: Plenum Press, 1984.

[18] R. S. Freund, R. C. Wetzel, R. J. Shul, and T. R. Hayes, "Cross-section measurements for electron impact ionization of atoms," Phys. Rev. A, vol. 41, pp. 3575-3595, 1990. 
[19] J. Lörinčík, Z. Šroubek, H. Eder, F. Aumayr, and H. Winter, "Kinetic electron emission from clean polycrystalline gold induced by impact of slow $\mathrm{C}^{+}, \mathrm{N}^{+}, \mathrm{O}^{+}$, $\mathrm{Ne}^{+}, \mathrm{Xe}^{+}$, and $\mathrm{Au}^{+}$ions," Phys. Rev. B, vol. 62, p. 16116, 2000. 


\section{Figure Captions}

Fig. 1 Illustration of the current delay, with the applied voltage as a parameters; applied voltage (top) and discharge current of a magnetron discharge (bottom); $\mathrm{Cu}$ target, $\mathrm{Ar} 1.3$ $\mathrm{Pa}, 100$ pulses per second.

Fig. 2 Overlay of ten pulses taken over a course of 30 minutes; the time zero is defined as the time when the voltage is applied. The onset of the current is delayed by over 100 $\mu$ s yet appears highly reproducible within an interval of $500 \mathrm{~ns}$. The voltage is stable within $\pm 1.5 \mathrm{~V}$.

Fig. 3 Delay of the current onset as a function of the applied voltage for different target materials; argon at $1.3 \mathrm{~Pa}$; voltage pulse duration $400 \mu \mathrm{s}, 100$ pulses per second.

Fig. 4 Delay of the discharge current as a function of the applied voltage for $\mathrm{Cu}$ and $\mathrm{Nb}$ targets with reactive process gases at $1.3 \mathrm{~Pa}$, though the curves for argon are included for comparison; voltage pulse duration $400 \mu \mathrm{s}, 100$ pulses per second.

Fig. 5 Delay of the current onset as (top) a function of the applied voltage with the argon pressure as a parameter for a zinc cathode and (bottom) as a function of the pressure with the voltage as a parameter, copper target; voltage pulse duration $400 \mu \mathrm{s}, 100$ pulses per second.

Fig. 6 Delay of the current onset (top) as a function of the pulse duration for constant repetition rate of 100 pulses per second, and (bottom) as a function of the pulse repetition rate for a constant pulse duration of $200 \mu \mathrm{s}$ (tungsten target and argon at $1.3 \mathrm{~Pa}$, applied voltage pulse of $600 \mathrm{~V}$ ).

Fig. 7 Replots of figures 3 (top), 4 (center), and top part of 5 (bottom) using the inverse of the delay as the variable of interest. 

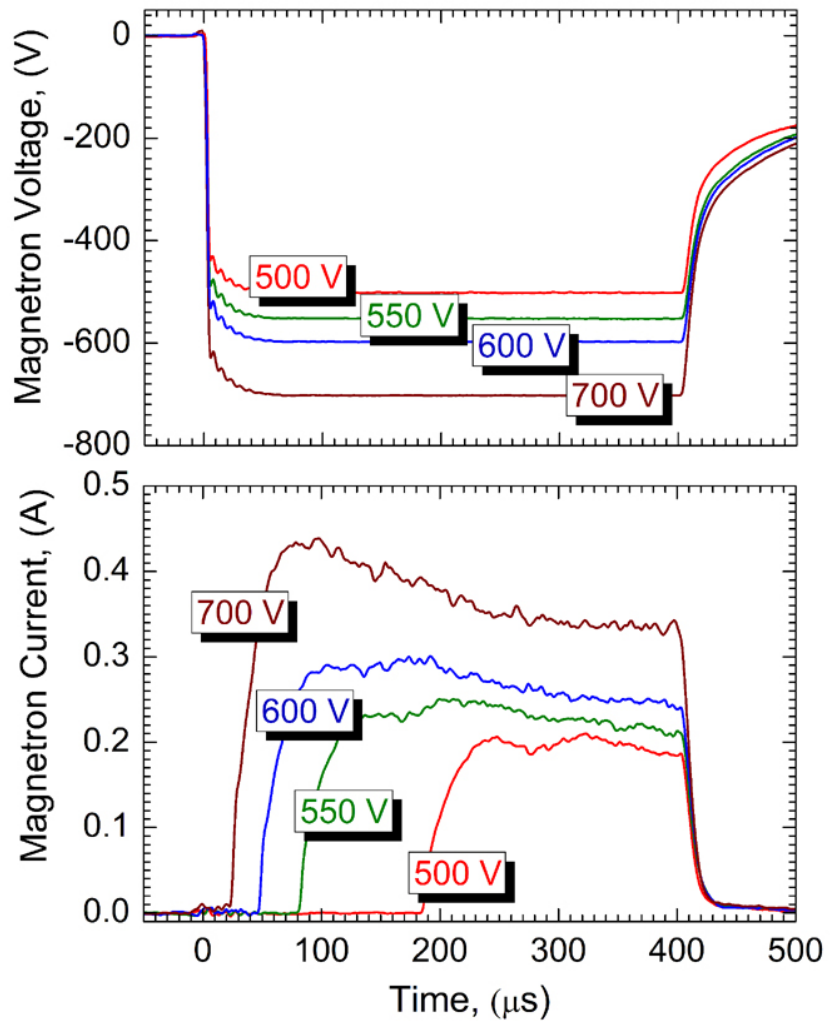

Fig. 1 


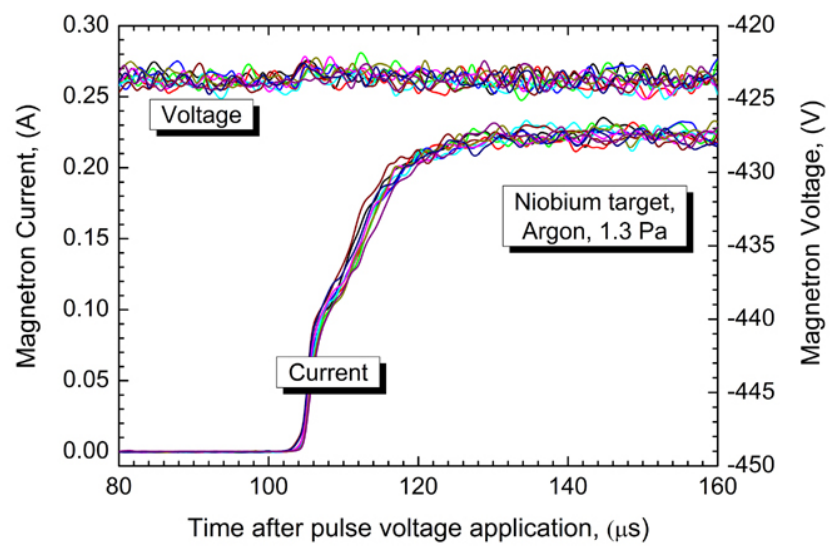

Fig. 2 


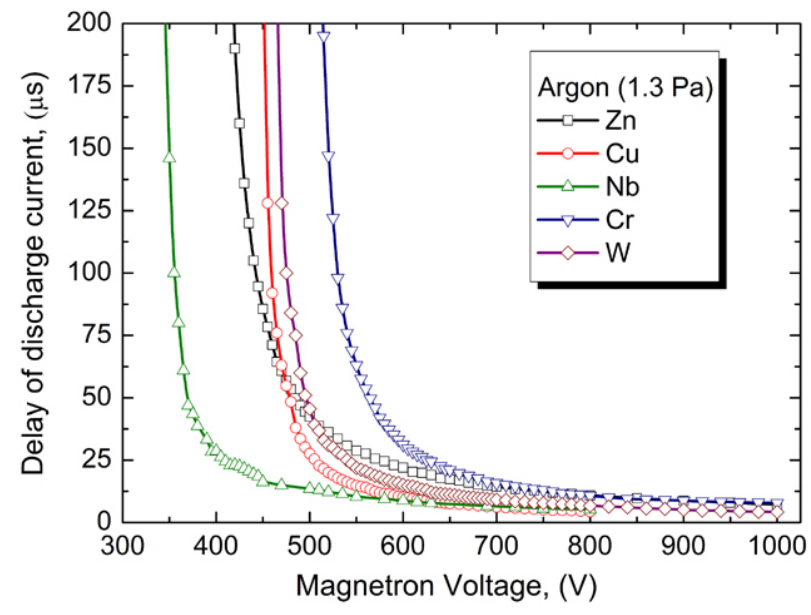

Fig. 3 


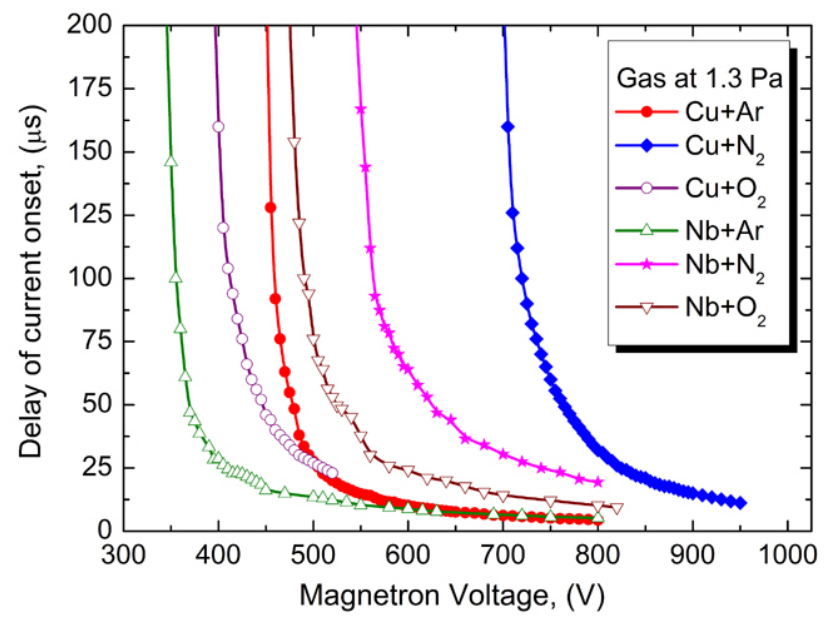

Fig. 4 

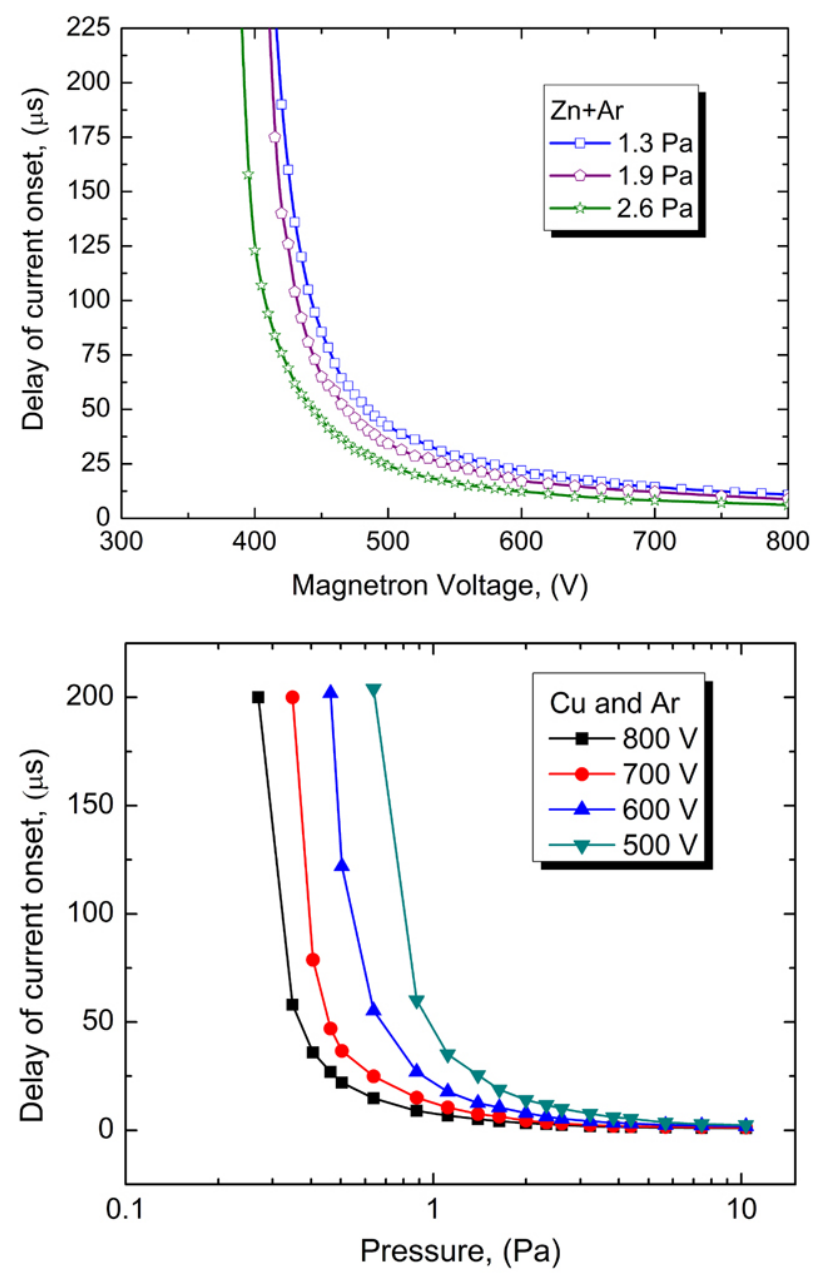

Fig. 5 

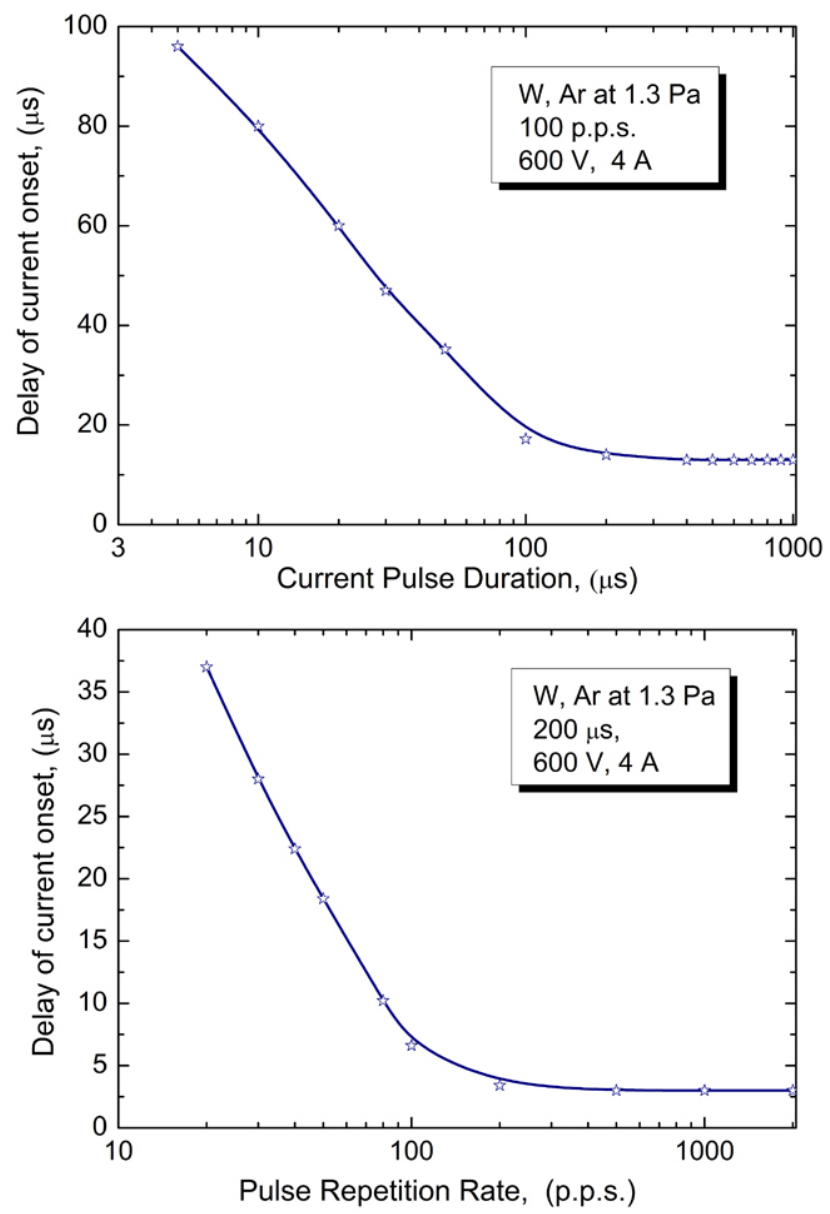

Fig. 6 

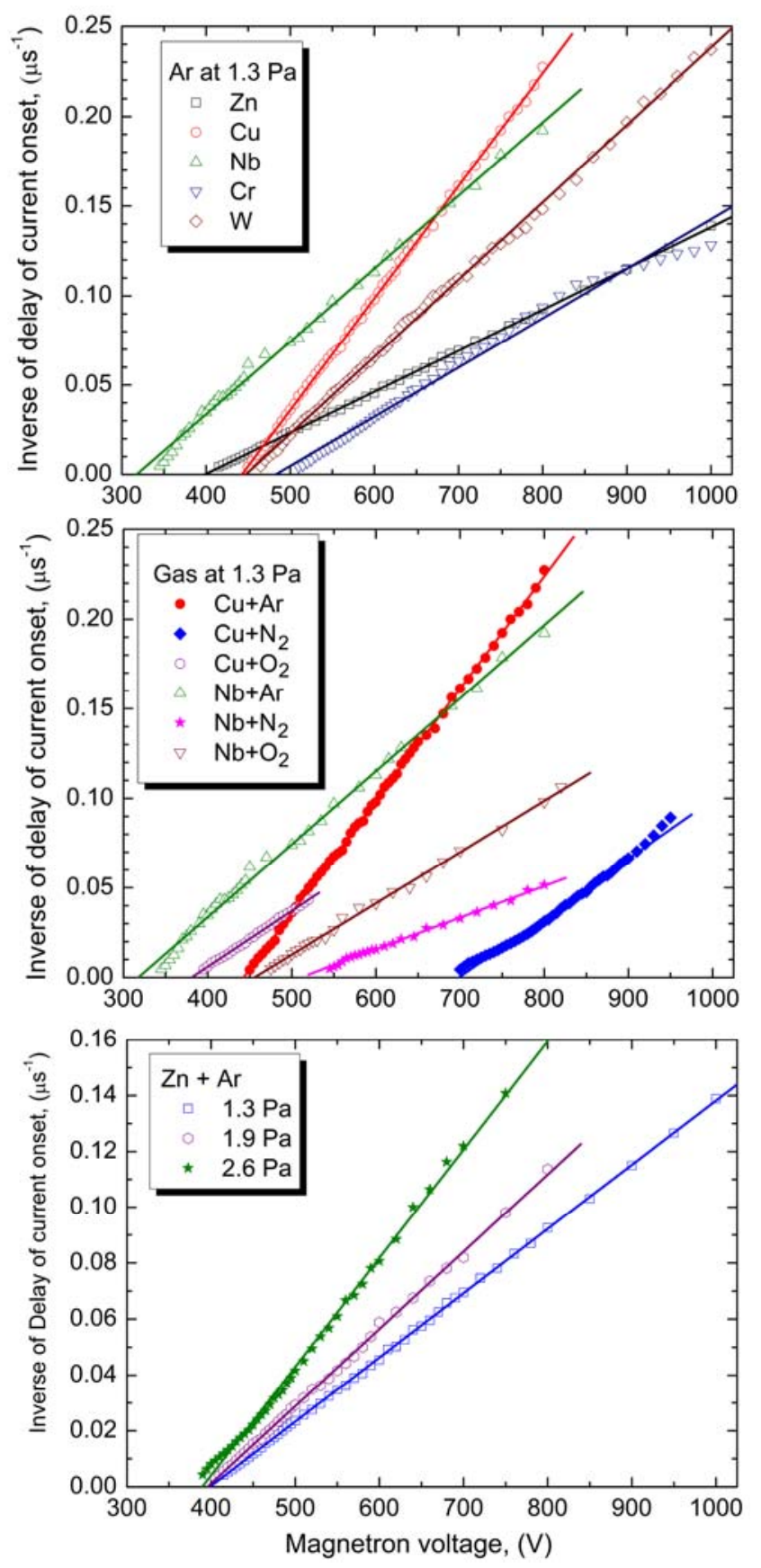

Fig. 7 


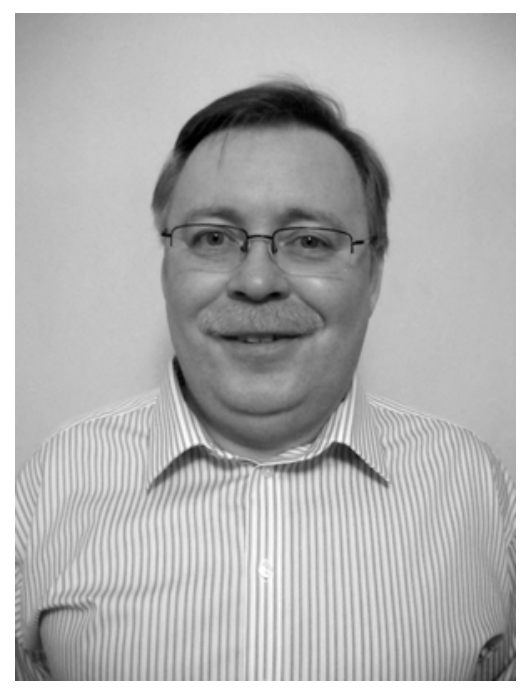

Georgy Yu. Yushkov is a Principal Scientist at the High Current Electronics Institute in Tomsk, Siberia, Russia, his native town. He obtained his M.Sc. degree at the Department of Nuclear Physics of the Tomsk Polytechnic University in 1988. The same year he joined the High Current Electronics Institute of the Russian Academy of Sciences. He received the Ph.D. degree in 1993 and the Dr. Sci. degree in 2002, both from the High Current Electronics Institute, for the study and development of ion sources and the investigation of plasmas. His field of research includes vacuum arc plasma and ion sources, vacuum arcs physics, high current glow, and ion emission from plasmas. He is author/co-author of about 100 articles in refereed scientific journals and his work has been recognized by several international and Russian awards. 


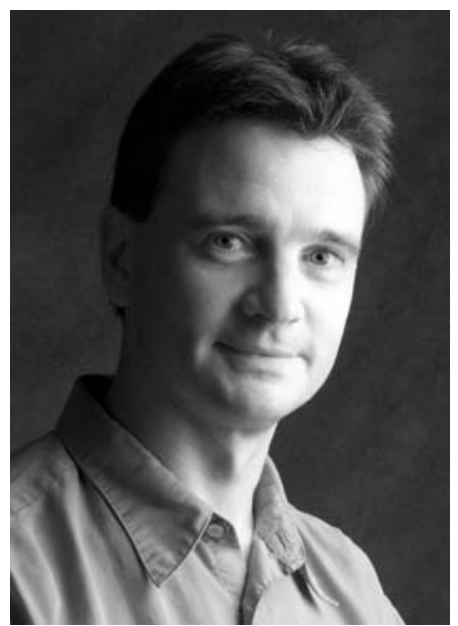

André Anders is a Senior Scientist and the Leader of the Plasma Applications Group at Lawrence Berkeley National Laboratory, Berkeley, California. After studying physics in Germany, Poland, and Russia, he worked on electrode phenomena at the Academy of Sciences in East Berlin (1987-1991). Since 1992 he is affiliated with Berkeley Lab performing research on a various plasma and materials issues. He is the author/editor three books including Cathodic Arcs: From Fractal Spots to Energetic Condensation, (Springer, NY, 2008). He is the author of more than 250 papers in peer-reviewed journals. He serves in several international conference committees including as Chairman of the Permanent International Scientific Committee of the International Symposia on Discharges and Electrical Insulation. He joined the Editorial Boards of Surface and Coatings Technology, Applied Physics Letters, and the Journal of Applied Physics, and recently became an Associate Editor of the Journal of Applied Physics. Dr. Anders was elected Fellow of the Institute of Electrical and Electronic Engineers (IEEE), the American Physical Society (APS), and the Institute of Physics (IoP). 